\title{
Detachment of Membrane Bound Virions by Competitive Ligand Binding Induced Receptor Depletion
}

\author{
Nagma Parveen, ${ }^{\dagger}$ Stephan Block, ${ }^{\dagger}$ Vladimir P. Zhdanov, ${ }^{\dagger, \dagger}$ Gustaf E. Rydell, ${ }^{\S}$ and Fredrik Höök ${ }^{*},^{\dagger}(\mathbb{0}$ \\ ${ }^{\dagger}$ Department of Physics, Chalmers University of Technology, Gothenburg, Sweden \\ *Boreskov Institute of Catalysis, Russian Academy of Sciences, Novosibirsk, Russia \\ ${ }^{\S}$ Department of Infectious Diseases, Sahlgrenska Academy, University of Gothenburg, Gothenburg, Sweden
}

\section{Supporting Information}

ABSTRACT: Multivalent receptor-mediated interactions between virions and a lipid membrane can be weakened using competitive nonpathogenic ligand binding. In particular, the subsequent binding of such ligands can induce detachment of bound virions, a phenomenon of crucial relevance for the development of new antiviral drugs. Focusing on the simian virus 40 (SV40) and recombinant cholera toxin $\mathrm{B}$ subunit (rCTB), and using (monosialotetrahexosyl)ganglioside (GM1) as their common receptor in a supported lipid bilayer (SLB), we present the first detailed investigation of this phenomenon by employing the quartz crystal microbalance with dissipation (QCM-D)

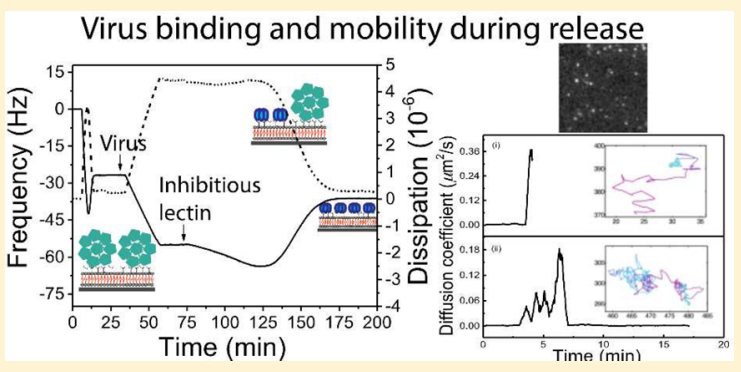
and total internal reflection fluorescence (TIRF) microscopy assisted $2 \mathrm{D}$ single particle tracking (SPT) techniques. Analysis of the QCM-D-measured release kinetics made it possible to determine the binding strength of a single SV40-GM1 pair. The release dynamics of SV40, monitored by SPT, revealed that a notable fraction of SV40 becomes mobile just before the release, allowing to estimate the distribution of SV40-bound GM1 receptors just prior to release.

\section{INTRODUCTION}

Prior to internalization and infection, virions first bind to specific receptors present on the external lipid membrane of their host cells. This process is controlled by the binding strength of single ligand-receptor pairs/bonds, the number of such pairs/bonds, and the ability to dynamically form and break these bonds. Since the binding strength of individual ligandreceptor pairs is often weak, ${ }^{1,2}$ establishment of multiple bonds is typically required to obtain a sufficient residence time for virus internalization to occur. ${ }^{3}$ The weak strength of the individual ligand-receptor pairs implies that the lateral interaction between them in solution is negligible, and accordingly their formation and rupture occur nearly independently or, in other words, in a sequential manner. Such random transitions between "states" of different underlying valencies can be described by the corresponding master equations for the number of ligand-receptor pairs (see section S4 in the Supporting Information and references therein) and are known to influence the receptor distribution on the cell membrane as well as the shape of membranes, ${ }^{3-8}$ factors that are believed to be crucial also during the internalization process. Insights regarding this as well as other multivalent biomolecular interactions can be gained by directly probing the kinetics of binding to cell membranes and mimics thereof $f^{9-12}$ or by titration studies with suspended binders displaying different valencies. ${ }^{1,4}$ Information on interaction kinetics can also be gained by time-resolved imaging of how single lipid vesicles transiently interact with surface-immobilized virions. ${ }^{9}$ However, the strong (power-law) dependence of the residence time on the number of ligand-receptor pairs makes the binding become irreversible already for a few bonds. High-resolution microscopy was therefore mostly used to track the spatial mobility of individual virus particles, thereby yielding information about their diffusivity which is determined by their multivalent interaction. ${ }^{13-15}$

A key motivation behind the above-mentioned basic studies aimed at unraveling the nature of the interaction between virions and a lipid membrane is to gain insights that can aid in the development of antiviral therapies. Numerous related applied studies were focused on attachment of inhibitors targeting the receptor-binding sites of (suspended or membrane-bound) virions, ${ }^{16-2}$ but the success of this approach in terms of approved drugs has been limited. An alternative stratergy is to target inhibitors toward the cellular receptors, thereby preventing attachment of virions to cell membranes. ${ }^{22-28}$ Despite fewer studies the latter approach has indeed offered a clinically used HIV antiviral, Maraviroc, which interacts with the cellular coreceptor CCR5, thereby inhibiting viral entry. ${ }^{29}$ Most of the corresponding assays ${ }^{22,23,25,28}$ were measured at steady-state conditions and various mechanistic aspects of the inhibition kinetics, ${ }^{26,27}$ such as inhibitor-induced transient changes in the interaction profile could therefore not be characterized. One interesting aspect of such kinetics, related to multivalency, is that in the course of inhibitor attachment the

Received: December 21, 2016

Revised: $\quad$ March 27, 2017

Published: March 28, 2017 
concentration of free receptors in a lipid membrane becomes reduced, and as a consequence, the number of receptor-ligand pairs for already bound virions may also decline, which will eventually result in detachment of these virions. Herein, we present what to our knowledge is the first study of this category.

As a model system to evaluate what type of information one can gain by measuring virus release induced by a competitive binder, we investigate how the addition of cholera toxin (CT) influences the release kinetics of simian virus (SV40) being prebound to a supported lipid bilayer (SLB) containing the glycosphingolipid (GSL) GM1, having the shared feature of being a receptor for both CT and SV40. ${ }^{5,30}$ As a model for SV40 we used a virus-like particle (VLP), which is a recombinant capsid containing 72 copies of the GM1-binding subunit VP1, known to display very similar binding behavior as native virions. ${ }^{5}$ To compete with the binding of SV40 to membrane-embedded GM1, we used the recombinantly expressed B subunit of cholera toxin ( $\mathrm{rCTB}$ ), which is responsible for the receptor binding and intracellular traficking of the toxin. ${ }^{31}$ rCTB forms a pentameric structure with a diameter around $6 \mathrm{~nm},{ }^{30}$ whereas the 72 pentameric VP1 units of the SV40 capsid form a symmetric spherical particle with a diameter of around $40 \mathrm{~nm}^{32}$ Despite lack of sequence homology, the structure of the VP1 pentamers making up SV40 is very similar to the $\mathrm{CTB}$ pentamer, each presenting five GM1 binding sites positioned with a distance of $3 \mathrm{~nm}$ between each other. ${ }^{33}$ The equilibrium dissociation constant of the monovalent interaction of single GM1 entities with these pentamers was previously determined using isothermal titration calorimetry to be on the order of a few $\mathrm{mM}$ for VP1 of SV40 ${ }^{1}$ and as low as $40 \mathrm{nM}$ for $\mathrm{CTB},{ }^{34}$ corresponding to Gibbs free energies of around $6 k_{\mathrm{B}} T$ and $17 k_{\mathrm{B}} T$, respectively. This significantly higher affinity between GM1 and $\mathrm{rCTB}$ compared to the interaction of GM1 with SV40, together with the positive cooperativity previously suggested for binding of the $\mathrm{rCTB}$ pentamer to multiple membrane-bound $\mathrm{GM}^{7,35}$ and its structural similarity with the VP1 pentamers, makes $\mathrm{rCTB}$ a suitable model compound to explore in the context of GM1mediated competitive binding with SV40 to a lipid membrane.

Quartz crystal microbalance with dissipation (QCM-D) monitoring was employed to follow rCTB-induced release kinetics of SV40 prebound to a SLB containing different GM1 concentrations, which combined with a kinetic model describing the multivalent SV40-GM1 and rCTB-GM1 interactions enabled an estimation of the strength of the former interaction when approaching a single bond. Complementary total internal reflection fluorescence (TIRF) microscopy experiments were focused on the influence of $\mathrm{rCTB}$ binding on the mobility of individual SV40 particles and aimed at determining the dynamic variation in number of receptors engaged in the binding during the release process. The results are conceptually valid in the broad context of GSLs and glycoconjugates being utilized as receptors by a large number of virions, such as polyomavirus, norovirus, rotavirus, parvovirus, and influenza virus ${ }^{36-38}$ as well as bacterial toxins and endogeneous lectins. ${ }^{37,39}$ This suggests in turn that the experimental and theoretical approach presented in this work will be generally applicable to unravel new insights regarding multivalent interactions for many different biological systems, thereby both aiding a fundamental understanding of virusreceptor interactions and offering an analytical foundation for the development of compounds aimed to inhibit virus infections by interfering with cell-surface receptors rather than the virus particles themselves.

\section{RESULTS AND DISCUSSION}

Release of Bound VLP by Subsequent rCTB Binding. SLBs with a total GM1 content ranging from 0.05 and 2 wt \% (which translates to 0.03 to $1.3 \mathrm{~mol} \%$ ) were formed by exposure of a silica-coated QCM-D crystal (placed in a cylindrical measurement cell with a diameter of $\sim 10 \mathrm{~mm}$ and height of $\sim 0.1 \mathrm{~mm}$ connected to a liquid handling system with a length of $\sim 120 \mathrm{~mm}$ and diameter of $\sim 1 \mathrm{~mm}$ ) to a mixture of vesicles made of pure 1-palmitoyl-2-oleoyl-sn-glycero-3-phosphocholine (POPC) lipids and vesicles made of POPC and 5 wt \% GM1 lipids (see section S1 C in the Supporting Information for the details of controlling the GM1 content in SLB). At 2 wt \% GM1, vesicle binding and subsequent rupture resulted in changes in resonance frequency, $f$, and energy dissipation, $D$, of $-27 \mathrm{~Hz}$ and $0.2 \times 10^{-6}$, respectively (Figure 1 ), in agreement with previous studies of SLB formation at

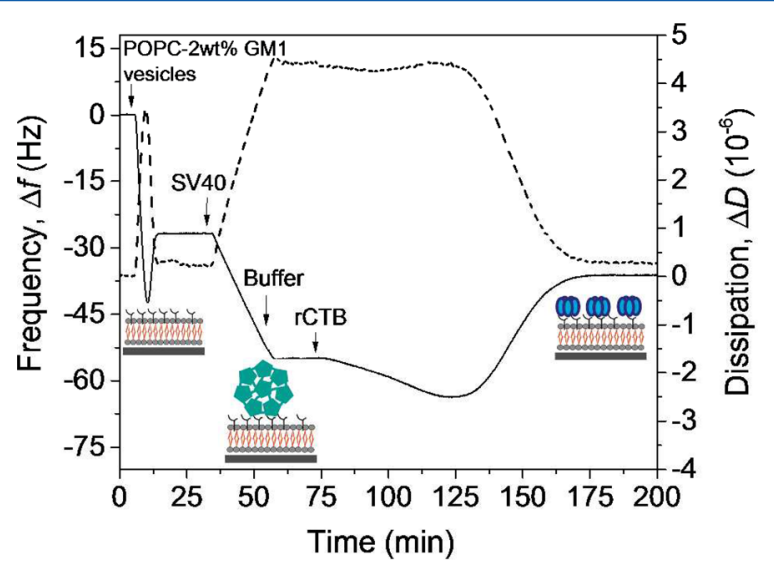

Figure 1. Time trace of QCM-D response (fifth overtone) upon SV40 and subsequent $\mathrm{rCTB}$ binding on a supported lipid bilayer (SLB) with 2 wt \% GM1. The solid and dotted lines correspond to the frequency and dissipation shifts. The measurements were performed by subsequently exposing the SLB with solutions of $0.139 \mathrm{nM}$ SV40, buffer, and $3.45 \mathrm{nM} \mathrm{rCTB}$ at a flow rate of $50 \mu \mathrm{L} / \mathrm{min}$. Arrows indicate the starting moments of solution exchange. Schematics of SLB with GM1, SV40 binding on the SLB, and finally rCTB binding followed by SV40 release are presented for better visualization of the competitive binding system. No binding was observed to bare POPC SLBs (data not shown).

these conditions. ${ }^{40}$ Subsequent binding of SV40 was performed by exposing the GM1-containing SLB to a SV40 suspension $(0.139 \mathrm{nM})$ for $20 \mathrm{~min}$ at a flow rate of $50 \mu \mathrm{L} / \mathrm{min}$, resulting in additional changes in $f$ and $D$ of $-28 \mathrm{~Hz}$ and $4.2 \times 10^{-6}$, respectively (Figure 1 ). This frequency shift is far from saturation, which was previously shown to correspond to around $-85 \mathrm{~Hz}$ using a higher SV40 concentration. ${ }^{8}$

Subsequent addition of $\mathrm{rCTB}(3.45 \mathrm{nM})$ at the same flow conditions resulted in an initial decrease in $f$ (mass uptake) which was followed by a dramatic increase (mass loss) that saturated at $-10 \mathrm{~Hz}$ (compared to the value corresponding to the original SLB) and a significant drop in $D$ that saturated at a value $(0.1 \pm 0.2) \times 10^{-6}$. The values measured after saturated binding of rCTB to a SV40-coated SLBs at different GM1 coverages (Figure $2 \mathrm{a}$ ) agree very well with the saturated shifts observed upon addition of $\mathrm{rCTB}$ to SLBs containing corresponding GM1 coverages but no SV40 (Figure S1), 

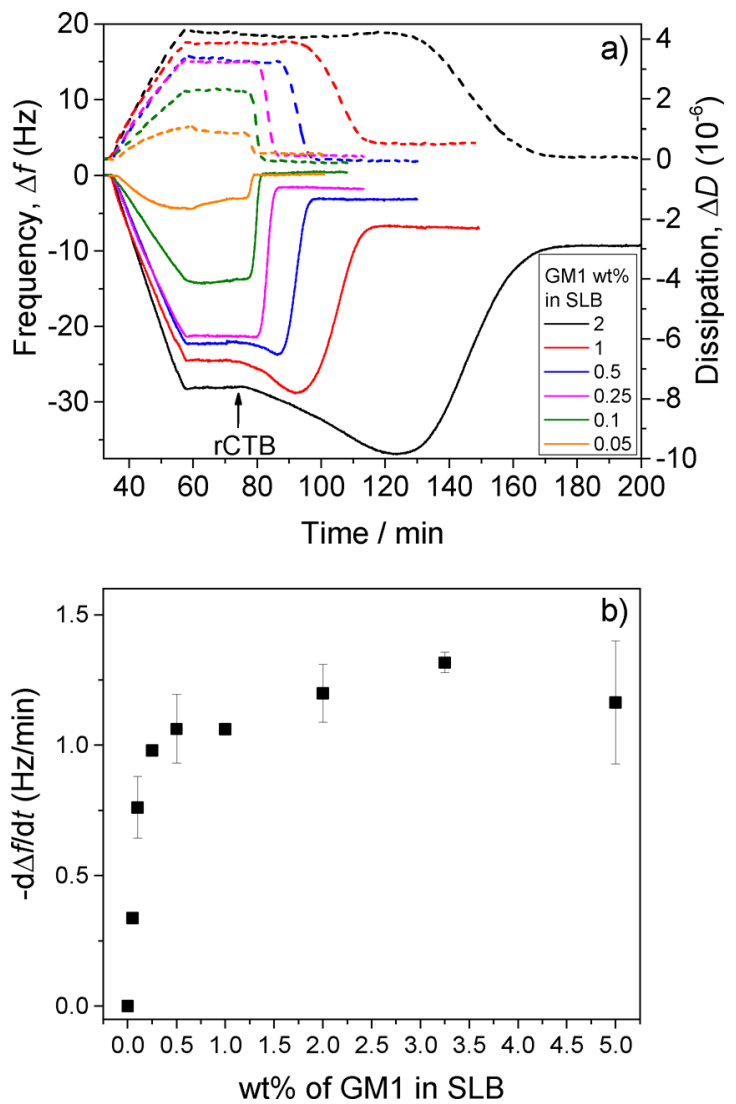

Figure 2. (a) QCM-D time trace of SV40 and subsequent $\mathrm{rCTB}$ binding on SLBs with different GM1 concentrations (0.05-2 wt \%) under bindng conditions that were otherwise the same as in Figure 1. The data are presented with an offset at the injection of the SV40 suspension. (b) Rate of frequency change upon SV40 binding versus the GM1 concentration in SLB. The error bars represent standard deviation of mean values determined from at least three data sets.

demonstrating that $\mathrm{rCTB}$ efficiently replaces SV40 from the SLB.

The rate of binding $(-\mathrm{d} \Delta f / \mathrm{d} t)$ of SV40 was observed to be nearly constant above and decrease dramatically below $0.25 \mathrm{wt}$ \% GM1 (Figure 2a,b) and displayed a nonlinear dependence on GM1 coverage.

This suggests that SV40 binding under these conditions is controlled globally by diffusion down to a GM1 content of $\sim 0.25$ wt $\%$, below which it becomes kinetically limited. The dramatic increase in the rate of binding between 0.05 and 0.25 wt \% further suggests that the formation of the first bond is not sufficient for the transition to the fully bound state; i.e., the rupture of the first bond just after its formation is more probable than the formation of a few additional bonds needed for firm binding. In other words, this means that the binding process is limited by the sequential formation of the first two or three bonds (accurate evaluation of this number is beyond our present goals although in principle it is possible via additional experiments and detailed simulations ${ }^{8}$ ). This interpretation is supported by detectable release of SV40 upon rinsing with buffer at GM1 coverages of 0.05 and 0.1 wt \%, while the binding was essentially irreversible at high GM1 coverage.

Inspecting the kinetics during the competitive phase indicates that the rate of $\mathrm{rCTB}$-induced SV40 release increased with decreasing GM1 content in the SLB (Figure 2a). In particular, the SV40 release at 0.05 wt \% GM1 occurs within the time of solution exchange in the QCM-D cell $(\sim 60 \mathrm{~s})$, while it takes several tens of minutes at 2 wt $\%$ GM1. The strong dependence of SV40 release kinetics on GM1 coverage cannot be attributed to a corresponding dependence of the rate of $\mathrm{rCTB}$ binding on GM1 coverage, which was in fact constant in this GM1 regime (Figure S1). A more plausible interpretation is thus that the binding avidity of SV40 decreases with decreasing GM1 content in SLB, indicating a smaller number of SV40-GM1 bonds at lower GM1 content.

Deconvolution of the Measured Frequency Response. To analyze rCTB-induced release kinetics of SV40 in further detail, one may note that both $\mathrm{rCTB}$ and SV40 contribute to $\Delta f$, while $\Delta D$ induced upon $\mathrm{rCTB}$ binding is negligible compared to that induced upon SV40 binding (Figure 1 and Figure S1). This makes it possible to deconvolute the measured (total) $\Delta f$ time trace into separate time traces for SV40 $\left(\Delta f_{\mathrm{SV} 40}\right)$ and $\operatorname{rCTB}\left(\Delta f_{\mathrm{rCTB}}\right)$, as shown e.g. in Figure 3a (see section S2 C in the Supporting Information for more information). This treatment of the data illustrates that SV40 release starts when the binding of $\mathrm{rCTB}$ approaches saturation $(\sim-10 \mathrm{~Hz})$, a behavior that was observed irrespective of GM1
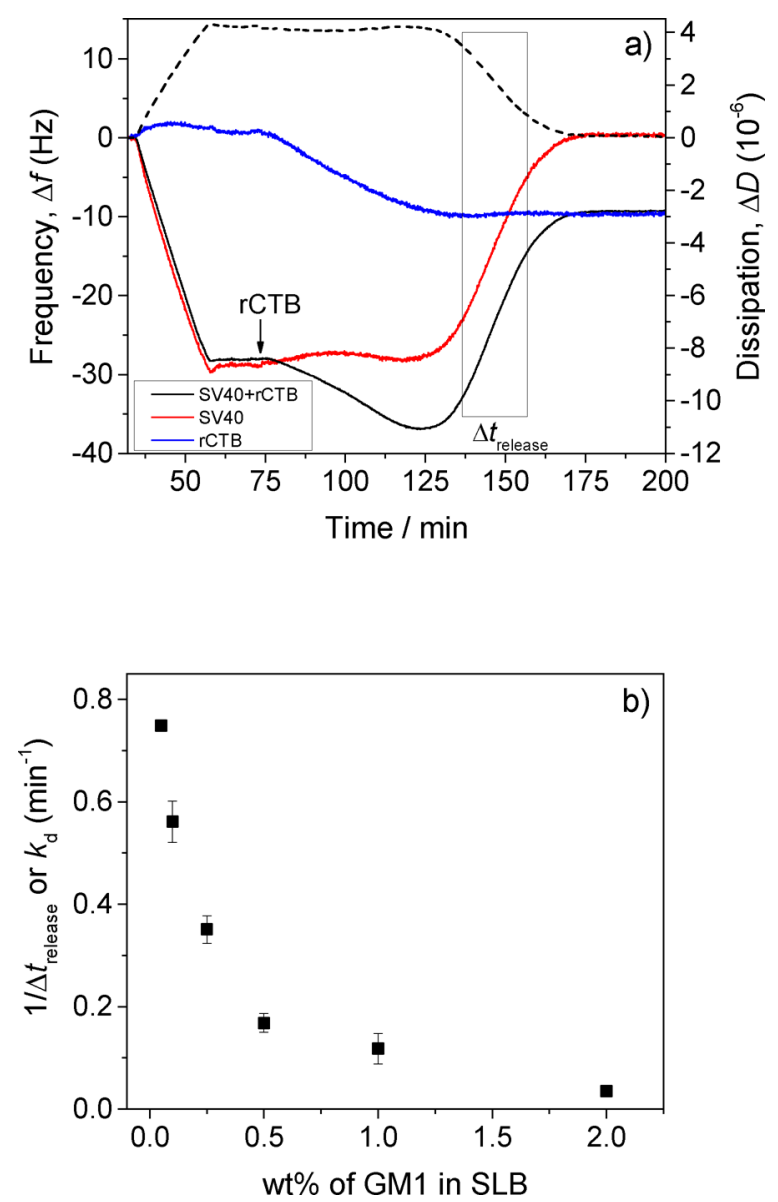

Figure 3. (a) Deconvolution of the measured frequency time trace at 2 wt \% GM1 in SLB: deconvoluted SV40 response (red line), deconvoluted rCTB response (blue line), measured dissipation response (dashed line). The SV40 release kinetics was essentially linear within the time interval $\Delta t_{\text {release }}$ (highlighted by the rectangle). (b) Inverse of the release time, $\Delta t_{\text {release }}$ decreases monotonously with increasing GM1 concentration in SLB and $1 / \Delta t_{\text {release }}$ approximately yields a release rate constant $k_{\mathrm{d}}$. The error bars represent standard deviation of mean values determined from at least three data sets. 
content. This thus demonstrates that SV40 release does not start until a majority of GM1 lipids are engaged in binding of rCTB; i.e., SV40 remains attached and is detected by QCM-D up to the stage when it has only a few (as already noted in the context of adsorption kinetics) or even a single (last) bond.

Quantification of the Binding Energy of VLP to GM1. For a more quantitative analysis of the release kinetics it is crucial to determine the surface coverage of GM1, SV40, and rCTB. The lipid membrane area corresponding to each GM1 is $\sim 1 \mathrm{~nm}^{2}$, and the extension of its hydrophilic headgroup above the SLB is $\sim 1 \mathrm{~nm} .{ }^{41}$ With an average radius of SV40 of $20 \mathrm{~nm}$ (Figure S3) and under the assumption that the SLB is nearly flat (this assumption appears to be reasonable because the membrane-support interaction is expected to prevent appreciable deformation of the SLB), an area of $125 \mathrm{~nm}^{2}$ of each SV40 can be reached by GM1, which corresponds to around 10 capsid proteins (VP1) of SV40 as detailed in section S4A of the Supporting Information (for virus binding to cells or giant vesicles, this number may be appreciably larger due to wrapping of a VLP or virion by the membrane ${ }^{5}$ ). Accordingly, since each capsid protein can potentially bind to a single GM1, a maximum of $10 \mathrm{GM} 1\left(n_{\mathrm{SV} 40}=10\right)$ can under these strict assumptions be accommodated within the contact area of SV40 and a flat SLB. From the difference in the measured frequency shift of POPC +2 wt \% GM1 and a pure POPC SLBs, the GM1 coverage, $c_{\mathrm{GM} 1}^{0}$, in each lipid layer of the SLB was determined to be $1.8 \times 10^{-2} \mathrm{~nm}^{-2}$ (here and below, "coverage" and "surface concentration" are used interchangeably), which matches well with previously reported estimates. ${ }^{7,40}$ Since a conversion of $\Delta f$ of QCM-D into coupled mass is not straightforward due to the influence of hydrodynamically coupled water to VLPs and $\mathrm{rCTB},{ }^{42}$ surface plasmon resonance (SPR) was used to quantify the molecular mass uptake of SV40 and $\mathrm{rCTB}$. With the latter technique, the surface coverage of SV40, $c_{\mathrm{SV} 40}$, was estimated to $2 \times 10^{-4} \mathrm{~nm}^{-2}$ at 2 wt $\%$ GM1 (Figure S2b in the Supporting Information). With $10 \mathrm{GMl}$ per SV40, the surface concentration of GM1 that is engaged in SV40 binding, $n_{\mathrm{SV} 40} c_{\mathrm{SV} 40}$, thus becomes $2 \times 10^{-3} \mathrm{~nm}^{-2}$ at $2 \mathrm{wt}$ $\%$, which is significantly lower than the total GM1 coverage $\left(c_{\mathrm{GM} 1}^{0}=1.8 \times 10^{-2} \mathrm{~nm}^{-2}\right)$. This means that the number of GM1 available for $\mathrm{rCTB}$ binding is essentially unaffected by the precence of SV40, which is consistent with the observation of nearly identical binding kinetics of $\mathrm{rCTB}$ in the presence (Figure 3) and absence of SV40 (Figure S1). The saturated surface coverage of $\mathrm{rCTB}$ at $2 \mathrm{wt} \% \mathrm{GM} 1$ was estimated using SPR to be $0.6 \times 10^{-2} \mathrm{~nm}^{-2}$ (Figure S2a), yielding a rCTB to GM1 ratio of $1: 2.8$. This ratio agrees well with previous reports of the number of receptors bound per rCTB molecule below 5 mol \% (7.5 wt \%) GM1 in the SLB. ${ }^{40}$

The estimates above were made from pure stoichiometric considerations, while in reality, the number of bonds that are engaged in the binding also depends on the probability of single bond formation. With $c_{\mathrm{SV} 40 / \mathrm{rCTB}}$ representing the surface coverage of either SV40 or rCTB and $c_{\mathrm{GM} 1}^{0}$ the total surface coverage of GM1, the balanced condition for the surface coverage of free/available GM1, $c_{\mathrm{GM} 1}$, can be expressed as

$$
c_{\mathrm{GMM} 1}+n_{\mathrm{SV} 40} P_{\mathrm{SV} 40} c_{\mathrm{SV} 40}+n_{\mathrm{rCTB}} P_{\mathrm{rCTB}} c_{\mathrm{rCTB}}=c_{\mathrm{GM} 1}^{0}
$$

where $P_{\mathrm{SV} 40 / \mathrm{rCTB}}$ is the probability that a given bond is formed and $n_{\mathrm{SV} 40 / \mathrm{rCTB}}$ the maximum number of bonds that each binding entity can form with GM1 lipids in the SLB (see section S4A in the Supporting Information). At the competitive conditions shown in Figures 2 and 3, the surface coverage of
rCTB determines $c_{\mathrm{GM}}$, and the latter determines the SV40 binding strength or, in other words, detachment rate of bound SV40. The formation and rupture of single SV40-GM1 and rCTB-GM1 bonds resulting in their redistribution during $\mathrm{rCTB}$ attachment are rapid because the binding energy of a single ligand-receptor bond/pair is weak. Hence, we can assume that the bond exchange is close to equilibrium and accordingly the probability of SV40-GM1 and rCTB-GM1 bond formation can be described as (derivation in section S4D in Supporting Information):

$$
P_{\mathrm{SV} 40 / \mathrm{rCTB}}=\frac{c_{\mathrm{GM} 1}}{K_{\mathrm{SV} 40 / \mathrm{rCTB}}+c_{\mathrm{GM} 1}}
$$

with

$$
K_{\mathrm{SV} 40 / \mathrm{rCTB}}=a^{-1} \exp \left(-E_{\mathrm{SV} 40 / \mathrm{rCTB}} / k_{\mathrm{B}} T\right)
$$

where $E_{\mathrm{SV} 40 / \mathrm{rCTB}}$ is the binding energy (Gibbs free energy for dissociation) of a single GM1-ligand pair of the respective particle and $a=1 \mathrm{~nm}^{-2}$ is the area comparable to the crosssection area of a single GM1.

As noted above, the SV40 release was observed at the late stage of the receptor redistribution, that is, when the $\mathrm{rCTB}$ adsorption was close to saturation (Figure 3a). At this stage, $c_{\mathrm{GM} 1}$ available for SV40 binding is, as already noticed, controlled by $\mathrm{rCTB}$ surface coverage and is according to the stoichiometric considerations presented above, expected to be appreciably smaller than $c_{\mathrm{GM} 1}^{0}$. Because of the low concentration of unbound GM1 at this stage, the reattachment of SV40 can be neglected, and the SV40 release kinetics can be represented as (derivation in section S4D in the Supporting Information):

$$
\frac{\mathrm{d} c_{\mathrm{SV} 40}}{\mathrm{~d} t}=-k_{\mathrm{d}} c_{\mathrm{SV} 40}
$$

where

$$
k_{\mathrm{d}}=k_{\mathrm{d} 1} P_{\mathrm{SV} 40}\left(1-P_{\mathrm{SV} 40}\right)^{n_{\mathrm{SV} 40}-1}
$$

is the effective release rate constant, expressed via $P_{\mathrm{SV} 40}$ and the rate constant, $k_{\mathrm{d} 1}$, of the rupture of the last bond accompanied by SV40 release. In this context, we repeat (cf. the Introduction) that the events of formation and rupture of SV40-GM1 bonds are sequential, and accordingly SV40 release occurs only after rupture of the last bond, although the sequential rupture of the last bonds may be below the acquisition rate of the measurements. Equation 5 is derived assuming that the redistribution of the number of bonds for each SV40 is close to equilibrium even for the last bond. In reality, due to SV40 detachment, the probability to have 1 or 2 bonds may be lower than that at equilibrium. The corresponding (cumbersome) modifications of the theoretical model are, however, not significant for our analysis and estimates.

In eq 5 the dependence of $k_{\mathrm{d}}$ on $P_{\mathrm{SV} 40}$ is strong because $n_{\mathrm{SV} 40}$ is relatively large. During the SV40 release, as already noticed, $P_{\mathrm{SV} 40}$ is controlled primarily by the surface coverage of $\mathrm{rCTB}$ and is almost constant because this phase is relatively short and $c_{\mathrm{rCTB}}$ was nearly constant over the release kinetics $\left(\Delta f_{\mathrm{rCTB}}\right.$ in Figure 3a). Under such circumstances, the release kinetics can be characterized by an effective first-order release rate constant which can be identified with $k_{\mathrm{d}}$ (eq 5). At 2 wt \% GM1, we have $k_{\mathrm{d}}=0.05 \mathrm{~min}^{-1}$ (Figure $2 \mathrm{~b}$ ). Using for this rate constant eq 5 in combination with eq 2 and employing $n_{\mathrm{SV} 40}=10$ (estimated in section S4A of the Supporting Information), $k_{\mathrm{d} 1}=10^{8} \mathrm{~s}^{-1}$ 
(estimated in section S4D of the Supporting Information), and $c_{\mathrm{GM} 1}=0.1 c_{\mathrm{GM} 1}^{0}=1.8 \times 10^{-3} \mathrm{~nm}^{-2}$ (estimated taking into account that release occurs at the late stage of $\mathrm{rCTB}$ binding where $\left.c_{\mathrm{GM} 1} \ll c_{\mathrm{GM} 1}^{0}\right)$, one obtains $P_{\mathrm{SV} 40}=0.945, K_{\mathrm{SV} 40}=1.0 \times$ $10^{-4} \mathrm{~nm}^{-2}$, and $E_{\mathrm{SV} 40}=9.2 k_{\mathrm{B}} T=22.3 \mathrm{~kJ} / \mathrm{mol}$. Note that quantitative estimates of this type at low GM1 content $(<0.5 \mathrm{wt}$ $\%)$ are not reliable because the assumption that $c_{\mathrm{GM} 1}$ is constant over the release kinetics does not appear to be valid (Figure S4).

The maximum possible number of the SV40-GM1 bonds, $n_{\mathrm{SV} 40}$, is the most crucial parameter in our estimation of $P_{\mathrm{SV} 40}$ and therefore $E_{\mathrm{SV} 40}$ as well. Our estimate of this number, $n_{\mathrm{SV} 40}$ $=10$ (section S4A in the Supporting Information), is based on the assumption that the SLB is perfectly flat. However, during the contact with SV40, the SLB can be locally deformed, and accordingly $n_{\mathrm{SV} 40}$ may be larger than 10 . In particular, Szklarczyk et al. ${ }^{8}$ recently reported that $n_{\mathrm{SV} 40}$ may be as high as 40. Repeating our estimates with the latter value of $n_{\mathrm{SV} 40}$, we obtain $P_{\mathrm{SV} 40}=0.488, K_{\mathrm{SV} 40}=1.9 \times 10^{-3} \mathrm{~nm}^{-2}$, and $E_{\mathrm{SV} 40}=$ $6.3 k_{\mathrm{B}} T=15.6 \mathrm{~kJ} / \mathrm{mol}$. With the binding energies $E_{\mathrm{SV} 40}=22.3$ and $15.6 \mathrm{~kJ} / \mathrm{mol}$ obtained here for $n_{\mathrm{SV} 40}=10$ and $n_{\mathrm{SV} 40}=40$, the corresponding monovalent GM1-SV40 dissociation constant becomes 0.1 and $2 \mathrm{mM}$, respectively. These numbers are in reasonable agreement with values reported for the monovalent interaction of GM1 with suspended SV40 (1-5 $\mathrm{mM})^{1}$ but also illustrate the importance of being able to accurately estimate $n_{\mathrm{SV} 40}$.

Mobility Determination from TIRFM Assay. To analyze the $\mathrm{rCTB}-\mathrm{SV} 40$ competition process in further detail and, more specifically, to gain additional information regarding the number of engaged GM1 linkers to SV40, we complemented the ensemble-averaged QCM-D measurements by SPT analysis of individual SV40 VLPs bound to a GM1-containing SLB (Figure 4). The SV40 VLPs were marked by dye-labeled

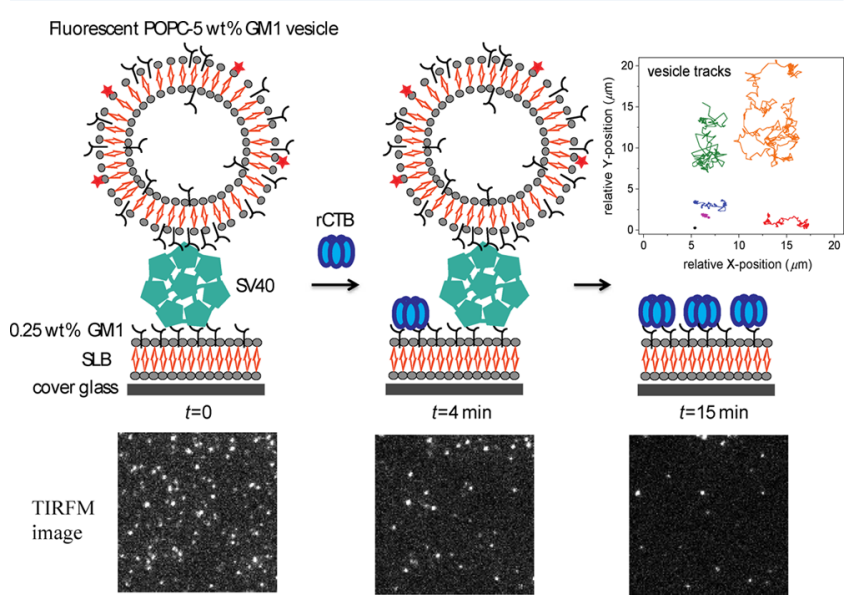

Figure 4. Illustration of tracking (SPT) measurements of individual SV40 VLPs using TIRF microscopy upon competitive binding induced by $\mathrm{rCTB}$ (illustration not to the scale). The dimension of each micrograph is $50 \mu \mathrm{m} \times 50 \mu \mathrm{m}$.

vesicles containing 5 wt \% GM1, which is much higher than the highest GM1 concentration used for SLB formation. As the release rate strongly decreases with increasing GM1 concentration (Figure $3 \mathrm{~b}$ ), this ensured firm vesicle-SV40 linking even under competition conditions (because the rate of SV40 release from the SLB was significantly higher than the release rate of dye-labeled vesicles from SV40 VLPs). The average diameter of the dye-labeled vesicles $(90 \mathrm{~nm})$ was not considerably larger than that of the VLPs $(40 \mathrm{~nm})$ which together with the low VLP coverage ensured attachment of single vesicle to single VLP and thereby qualifies the criteria of single VLP detection. ${ }^{9}$ SV40 attachment and release (Figure 4, from left to right) were monitored by TIRFM (see Movie S1), allowing to extract single virion trajectories until release of the tracked virion occurred (see Figure 4, insets, for representative trajectories).

Interestingly, for a GM1 concentration of $0.25 \mathrm{wt} \%$ in the SLB, all SV40 VLPs were essentially immobile directly after attachment (showing an apparent diffusion coefficient $D$ of $0.002 \mu \mathrm{m}^{2} / \mathrm{s}$, attributed to originate primarily from localization noise during the tracking process). However, after a lag time of rCTB addition we observed four different types of trajectories (Figure 5a): (i) VLPs that remained immobile directly prior to release, (ii) VLPs that became mobile prior to release, (iii) VLPs that became mobile, but turned immobile again and showed no release, and (iv) VLPs that remained immobile during the whole TIRF measurement, showing no release at all. These remarkable observations indicate that the SV40 release process is more complex than suggested by QCM-D (providing ensemble-averaged results) and that interaction with $\mathrm{rCTB}$ does not necessarily result in direct release of all bound SV40 (see case (iii) in Figure 5a). To better understand the observed SPT dynamics and its relation to the ensemble-averaged kinetics, we calculated the temporal change of the number of bound SV40 VLPs, which showed (after a short lag period) a sharp decrease followed by a slow transition to a constant SV40 coverage (Figure $5 \mathrm{~b}$, black line). A direct comparison with the QCM-D results (Figure $5 b$, red line), obtained using the same GM1 content in the SLB and $\mathrm{rCTB}$ concentration, shows good agreement of the release dynamics for both complementary approaches, confirming our previous interpretation of the QCM-D data. In particular, the fraction of VLPs which do not detach in the SPT analysis, and which could not be accurately determined from the QCM-D analysis, is relatively small $(\sim 8 \%)$, and their presence is not crucial for the analysis of the ensemble-averaged kinetics.

In a next step, we determined the $D$ distributions of SV40 VLPs directly after injection of $\mathrm{rCTB}$ and prior to release of the VLPs (Figure 5c) by calculating for each observed SV40 trajectory the $D$ value from the data of the first $10 \mathrm{~s}$ after $\mathrm{rCTB}$ addition or of the trajectory's last $10 \mathrm{~s}$, respectively, and by finally compiling all these $D$ values into histograms. Directly after rCTB injection, most of the SV40 VLPs are immobile, indicated by a single peak at $0.002 \mu \mathrm{m}^{2} / \mathrm{s}(\log D=-2.7)$ in the respective $D$ histogram (Figure $5 c$ ), which is attributed to localization noise and marks the lower resolution limit in $D$ determination. Prior to the release, a mobile peak appears at 0.1 $\mu \mathrm{m}^{2} / \mathrm{s}(\log D=-1)$ in addition to the immobile peak, indicating that approximately $25 \%$ of the VLPs become mobile (due to rCTB competition lowering the number of SV40GM1 bonds) before releasing, while approximately $75 \%$ of the VLPs appear to release directly from an immobile state.

The coefficient of diffusion of SV40 VLPs, which become mobile after rCTB addition, depends on the number of bound GM1 receptors, $n$, at a particular time. Our recent experimental studies $^{13}$ of a similar system (receptor-limited diffusion of lipid vesicles) indicate that the corresponding dependence is expected to be $D=D_{\mathrm{GM} 1} / n$, where $D_{\mathrm{GM} 1}$ is the diffusion coefficient of a single GM1 receptor, and accordingly we have

$$
n=D_{\mathrm{GM} 1} / D
$$



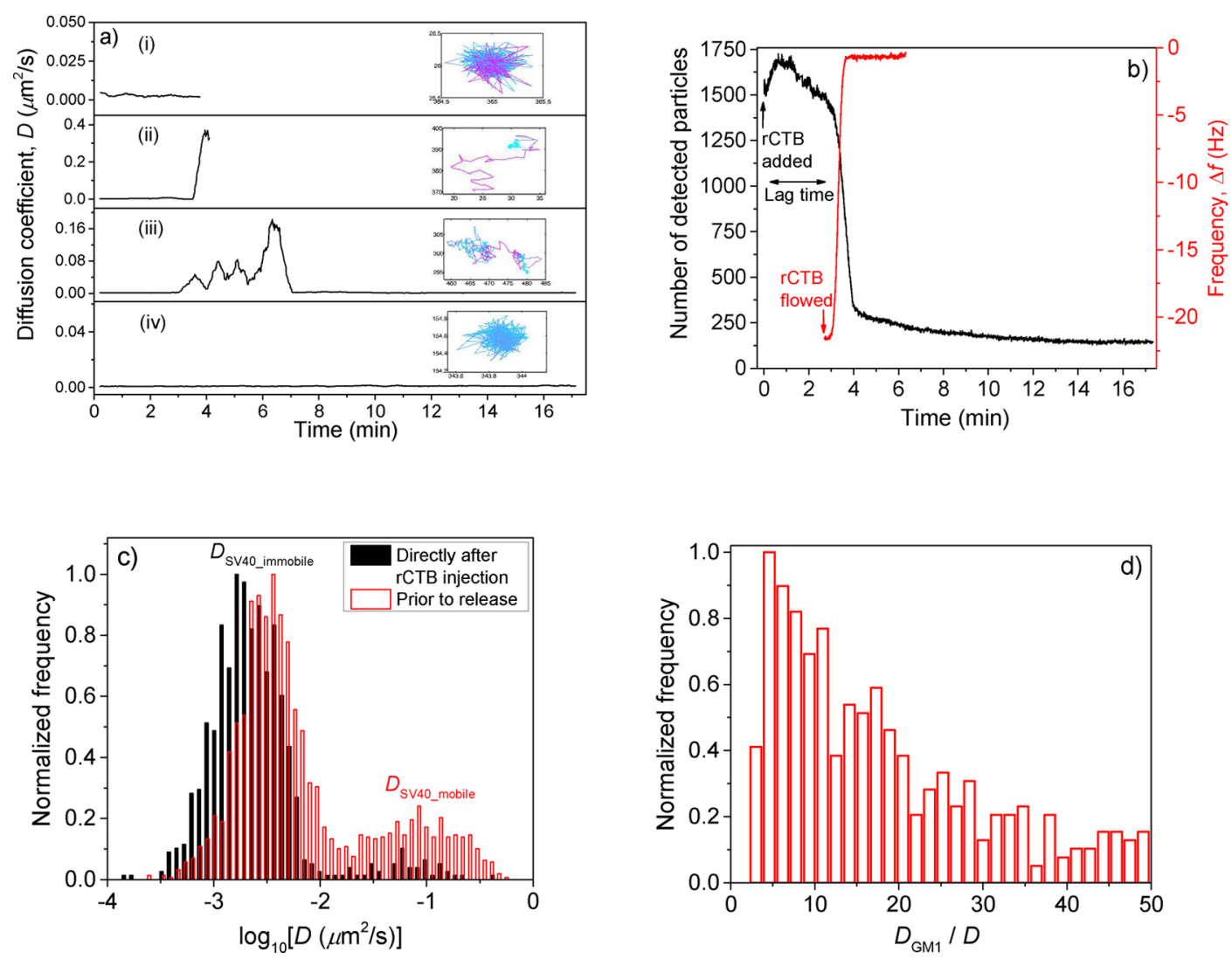

Figure 5. Mobility of SV40 VLPs changes due to competition with rCTB. (a) Four different modes [(i)-(iv)] of SV40 movement were observed using SPT (and further described in the text). The minimum and maximum values of the respective diffusion coefficient are employed to designate the color gradient from cyan to magenta for each track. (b) SV40 coverage (determined using TIRFM, black, and QCM-D, red) in dependence of the observation time (after injecting $\mathrm{rCTB}$ ). Note that the SV40 concentration during VLP incubation differs between both techniques $(0.00695 \mathrm{nM}$ in TIRFM versus $0.139 \mathrm{nM}$ in QCM-D), while SLB GM1 concentration ( $0.25 \mathrm{wt} \%)$ and rCTB concentration $(34.5 \mathrm{nM})$ are the same in both cases. The shorter lag time in QCM-D data than in TIRF data is due to flow vs stagnant condition in the respective experimental setup. (c) Distribution of diffusion coefficient, $D$, of SV40 VLPs directly after rCTB injection and prior to the release of the VLPs triggered by competition with injected rCTB, which is converted into (d) the number of bonds formed between SV40 VLPs and GM1 receptors in the SLB prior to release as estimated by using eq 6 with $D_{\mathrm{GM} 1}=1.3 \mu \mathrm{m}^{2} / \mathrm{s}$. Here we show the $D_{\mathrm{GM} 1} / D$ distribution only for $n<50$, although it exhibits a small tail beyond this value. This was motivated by the observation that the mobile and immobile peaks start to overlap in this region, which introduces a notable bias for $D_{\mathrm{GM}} / D$ exceeding 50 .

Using this expression and taking into account that $D_{\mathrm{GM} 1}$ is in the range between 0.8 and $1.8 \mu \mathrm{m}^{2} / \mathrm{s}$, ${ }^{8,43}$ we roughly estimated the distribution of the number of GM1 receptors bound to SV40 VLPs directly prior to release (Figure 5d, using average $D_{\mathrm{GM} 1}=1.3 \mu \mathrm{m}^{2} / \mathrm{s}$ ). This distribution is skewed and relatively wide with a peak maxima around 6 , which is close to our geometric estimation of maximum number of contact points for SV40 on a planar SLB $\left(n_{\mathrm{SV} 40}=10\right)$. However, it also exhibits a tail with values $n>40$, which can be explained by either local SLB deformation upon VLP binding, local GM1 clustering into domains, crowding effects at high $\mathrm{rCTB}$ coverage, and/or limitations of eq 6 for very high $n$. Still, these results, supplemented with the literature, suggest that even at competition the number of SV40-GM1 pair can be up to 40; therefore, prior to competition it is likely to be 40 or more, thus rendering the VLPs immobile, and yields a value of the dissociation constant for the monovalent interaction $(>2 \mathrm{mM})$ in agreement with previous estimates. ${ }^{1}$ More interestingly, a sharp cutoff at $n=3$ is observed, indicating that with our temporal resolution the SV40 binding can be tracked down to this low $n$ value. This cutoff value matches well with the theoretical estimation ${ }^{8}$ of minimum number of SV40-GM1 contacts required for detecting SV40 with our temporal resolution, while analysis of VLPs with $n<3\left(D>0.4 \mu \mathrm{m}^{2} /\right.$ s) would require higher acquisition rates.

\section{CONCLUSIONS}

We can summarize our key findings as follows:

First, we observed and scrutinized competitive binding of rCTB and SV40 toward their common receptor, i.e., GM1 on cell membrane mimics using QCM-D. Because of the higher affinity of $\mathrm{rCTB}$ to GM1, its attachment results in redistribution of GM1 bound to $\mathrm{rCTB}$ and SV40 with subsequent release of SV40. This process was interpreted by using a theoretical model to determine the binding affinity of SV40 from its release kinetics, yielding values matching previous estimates using monovalent binders.

Second, our SPT analysis confirms and extends the ensemble-averaged QCM-D data. In particular, it explicitly shows that the attachment of $\mathrm{rCTB}$ induces $2 \mathrm{D}$ diffusion of SV40 prior to release (the diffusion coefficient increases by 5to 150 -fold), while a small fraction of SV40 does not release. In addition, the SPT analysis allows us to conclude that the bound SV40 can be observed even if the number of the SV40-GM1 bonds is reduced down to 3 , which in agreement with a recent modeling approach of the SV40-GM1 interaction. ${ }^{8}$ All these 
SPT results are inherently related to the $\mathrm{rCTB}$ and SV40 competition for receptors because the dynamics of $\mathrm{SV} 40^{15}$ and many other viruses on cell membrane mimics under other conditions are typically too slow to be tracked using traditional microscopes. ${ }^{14,15}$ Further, attempts to lower the receptor concentration for SPT measurements down to that corresponding to the regime where mobility should be observed typically result in too weak affinity to enable sufficient statistics. Therefore, our SPT setup is an unique approach to quickly follow the complex binding-unbinding dynamics of viruses on cell mimics or membranes and even to detect or further modify the receptor distribution specifically on cell membranes.

Finally, we note that the competition between virions and inhibitors (ligands) for receptors like we observed and scrutinized in our study can potentially be used in application related to antiviral therapies. In real systems an additional challenge of this lectin-based competitive approach will be cellular internalization of the inhibitor (ligand)-receptor complex, which is likely to replenish the receptors on cell surfaces, although both internalization ${ }^{44}$ and receptor regeneration ${ }^{45,46}$ processes are slow on the time scale of the kinetics of our system. The potential solution of this problem can be the design of inhibitors with no or slow internalization, such as mutated ligands with lower valency or greater distance between their binding sites than that of the wild types. ${ }^{4,47}$

\section{ASSOCIATED CONTENT}

\section{S Supporting Information}

The Supporting Information is available free of charge on the ACS Publications website at DOI: 10.1021/acs.langmuir.6b04582.

Additional materials and methods, preparation details, and supporting QCM-D and SPR, TIRFM experiments, and the theoretical background (PDF)

Movie S1 (AVI)

\section{AUTHOR INFORMATION}

\section{Corresponding Author}

*E-mail: fredrik.hook@chalmers.se (F.H.).

\section{ORCID}

Fredrik Höök: 0000-0003-1994-5015

\section{Present Address}

S.B.: Department of Chemistry and Biochemistry, Freie Universität Berlin, Berlin, Germany.

\section{Author Contributions}

N.P. did the QCM-D and microscopy experiments; V.P.Z. developed the theoretical analysis; N.P. and S.B. analyzed the experimental data; N.P., S.B., and F.H. conceived the project and wrote the paper together with V.P.Z.; G.E.R. conceived part of the study and assisted in the interpretation of the data and in the writing of the manuscript. All authors have given approval to the final version of the manuscript.

\section{Funding}

The authors thank the Knut and Alice Wallenberg Foundation (2012.0055), the Swedish Research Council (2014-5557), and the Swedish Foundation for Strategic Research (RMA11-0104 and SB12-0088) for funding.

\section{Notes}

The authors declare no competing financial interest.

\section{REFERENCES}

(1) Neu, U.; Woellner, K.; Gauglitz, G.; Stehle, T. Structural basis of GM1 ganglioside recognition by simian virus 40. Proc. Natl. Acad. Sci. U. S. A. 2008, 105 (13), 5219-5224.

(2) Zhdanov, V. P. Physical aspects of the initial phase of endocytosis. Phys. Rev. E 2013, 88 (6), 064701.

(3) Mammen, M.; Choi, S. K.; Whitesides, G. M. Polyvalent interactions in biological systems: Implications for design and use of multivalent ligands and inhibitors. Angew. Chem., Int. Ed. 1998, 37 (20), 2754-2794.

(4) Arnaud, J.; Trondle, K.; Claudinon, J.; Audfray, A.; Varrot, A.; Romer, W.; Imberty, A. Membrane deformation by neolectins with engineered glycolipid binding sites. Angew. Chem., Int. Ed. 2014, 53 (35), 9267-9270.

(5) Ewers, H.; Romer, W.; Smith, A. E.; Bacia, K.; Dmitrieff, S.; Chai, W. G.; Mancini, R.; Kartenbeck, J.; Chambon, V.; Berland, L.; Oppenheim, A.; Schwarzmann, G.; Feizi, T.; Schwille, P.; Sens, P.; Helenius, A.; Johannes, L. GM1 structure determines SV40-induced membrane invagination and infection. Nat. Cell Biol. 2010, 12 (1), 1118.

(6) Hammond, A. T.; Heberle, F. A.; Baumgart, T.; Holowka, D.; Baird, B.; Feigenson, G. W. Crosslinking a lipid raft component triggers liquid ordered-liquid disordered phase separation in model plasma membranes. Proc. Natl. Acad. Sci. U. S. A. 2005, 102 (18), $6320-6325$.

(7) Shi, J. J.; Yang, T. L.; Kataoka, S.; Zhang, Y. J.; Diaz, A. J.; Cremer, P. S. GM(1) clustering inhibits cholera toxin binding in supported phospholipid membranes. J. Am. Chem. Soc. 2007, 129 (18), 5954-5961.

(8) Szklarczyk, O. M.; Gonzalez-Segredo, N.; Kukura, P.; Oppenheim, A.; Choquet, D.; Sandoghdar, V.; Helenius, A.; Sbalzarini, I. F.; Ewers, H. Receptor concentration and diffusivity control multivalent binding of SV40 to membrane bilayers. PLoS Comput. Biol. 2013, 9 (11), e1003310.

(9) Bally, M.; Gunnarsson, A.; Svensson, L.; Larson, G.; Zhdanov, V. P.; Hook, F. Interaction of single viruslike particles with vesicles containing glycosphingolipids. Phys. Rev. Lett. 2011, 107 (18), 188103.

(10) Hidari, K.; Shimada, S.; Suzuki, Y.; Suzuki, T. Binding kinetics of influenza viruses to sialic acid-containing carbohydrates. Glycoconjugate J. 2007, 24 (9), 583-590.

(11) Nasir, W.; Nilsson, J.; Olofsson, S.; Bally, M.; Rydell, G. E. Parvovirus B19 VLP recognizes globoside in supported lipid bilayers. Virology 2014, 456, 364-369.

(12) Rydell, G. E.; Dahlin, A. B.; Hook, F.; Larson, G. QCM-D studies of human norovirus VLPs binding to glycosphingolipids in supported lipid bilayers reveal strain-specific characteristics. Glycobiology 2009, 19 (11), 1176-1184.

(13) Block, S.; Zhdanov, V. P.; Hook, F. Quantification of multivalent interactions by tracking single biological nanoparticle mobility on a lipid membrane. Nano Lett. 2016, 16 (7), 4382-4390.

(14) Ewers, H.; Jacobsen, V.; Klotzsch, E.; Smith, A. E.; Helenius, A.; Sandoghdar, V. Label-free optical detection and tracking of single virions bound to their receptors in supported membrane bilayers. Nano Lett. 2007, 7 (8), 2263-2266.

(15) Kukura, P.; Ewers, H.; Muller, C.; Renn, A.; Helenius, A.; Sandoghdar, V. High-speed nanoscopic tracking of the position and orientation of a single virus. Nat. Methods 2009, 6 (12), 923-927.

(16) Bovin, N. V.; Tuzikov, A. B.; Chinarev, A. A.; Gambaryan, A. S. Multimeric glycotherapeutics: New paradigm. Glycoconjugate J. 2004, 21 (8-9), 471-478.

(17) Cecioni, S.; Imberty, A.; Vidal, S. Glycomimetics versus multivalent glycoconjugates for the design of high affinity lectin ligands. Chem. Rev. 2015, 115 (1), 525-561.

(18) Goodfellow, I. G.; Evans, D. J.; Blom, A. M.; Kerrigan, D.; Miners, J. S.; Morgan, B. P.; Spiller, O. B. Inhibition of coxsackie B virus infection by soluble forms of its receptors: Binding affinities, altered particle formation, and competition with cellular receptors. $J$. Virol. 2005, 79 (18), 12016-12024. 
(19) Mammen, M.; Dahmann, G.; Whitesides, G. M. Effective inhibitors of hemagglutination by influenza-virus synthesized from polymers having active ester groups - insight into mechanism of inhibition. J. Med. Chem. 1995, 38 (21), 4179-4190.

(20) Matrosovich, M. N. Towards the development of antimicrobial drugs acting by inhibition of pathogen attachment to host-cells - a need for polyvalency. FEBS Lett. 1989, 252 (1-2), 1-4.

(21) Waldmann, M.; Jirmann, R.; Hoelscher, K.; Wienke, M.; Niemeyer, F. C.; Rehders, D.; Meyer, B. A nanomolar multivalent ligand as entry inhibitor of the hemagglutinin of avian influenza. J. Am. Chem. Soc. 2014, 136 (2), 783-788.

(22) Chen, Q.; Guo, Y. Influenza viral hemagglutinin peptide inhibits influenza viral entry by shielding the host receptor. ACS Infect. Dis. 2016, 2 (3), 187-193.

(23) Dorr, P.; Westby, M.; Dobbs, S.; Griffin, P.; Irvine, B.; Macartney, M.; Mori, J.; Rickett, G.; Smith-Burchnell, C.; Napier, C.; Webster, R.; Armour, D.; Price, D.; Stammen, B.; Wood, A.; Perros, M. Maraviroc (UK-427,857), a potent, orally bioavailable, and selective small-molecule inhibitor of chemokine receptor CCR5 with broadspectrum anti-human immunodeficiency virus type 1 activity. Antimicrob. Agents Chemother. 2005, 49 (11), 4721-4732.

(24) Dragic, T.; Trkola, A.; Thompson, D. A. D.; Cormier, E. G.; Kajumo, F. A.; Maxwell, E.; Lin, S. W.; Ying, W. W.; Smith, S. O.; Sakmar, T. P.; Moore, J. P. A binding pocket for a small molecule inhibitor of HIV-1 entry within the transmembrane helices of CCR5. Proc. Natl. Acad. Sci. U. S. A. 2000, 97 (10), 5639-5644.

(25) Kondru, R.; Zhang, J.; Ji, C.; Mirzadegan, T.; Rotstein, D.; Sankuratri, S.; Dioszegi, M. Molecular interactions of CCR5 with major classes of small-molecule anti-HIV CCR5 antagonists. Mol. Pharmacol. 2007, 73 (3), 789-800.

(26) Martinez-Avila, O.; Hijazi, K.; Marradi, M.; Clavel, C.; Campion, C.; Kelly, C.; Penades, S. Gold manno-glyconanoparticies: multivalent systems to block HIV-1 gp120 binding to the lectin DC-SIGN. Chem. Eur. J. 2009, 15 (38), 9874-9888.

(27) Patching, S. G. Surface plasmon resonance spectroscopy for characterisation of membrane protein-ligand interactions and its potential for drug discovery. Biochim. Biophys. Acta, Biomembr. 2014, 1838 (1), 43-55.

(28) Tabarani, G.; Reina, J. J.; Ebel, C.; Vives, C.; Lortat-Jacob, H.; Rojo, J.; Fieschi, F. Mannose hyperbranched dendritic polymers interact with clustered organization of DC-SIGN and inhibit gp120 binding. FEBS Lett. 2006, 580 (10), 2402-2408.

(29) De Clercq, E.; Li, G. Approved antiviral drugs over the past 50 years. Clin. Microbiol. Rev. 2016, 29 (3), 695-747.

(30) Holmgren, J.; Lonnroth, I.; Svennerh, L. Tissue receptor for cholera exotoxin - postulated structure from studies with gm1 ganglioside and related glycolipids. Infect. Immun. 1973, 8 (2), 208214.

(31) Claudinon, J.; Rydell, G. E.; Römer, W. Microbial toxins as tools in cell biology. In Bacterial Toxins: Genetics, Cellular Biology and Practical Applications; Proft, T., Ed.; Caister Academic Press: 2013.

(32) Kosukegawa, A.; Arisaka, F.; Takayama, M.; Yajima, H.; Kaidow, A.; Handa, H. Purification and characterization of virus-like particles and pentamers produced by the expression of SV40 capsid proteins in insect cells. Biochim. Biophys. Acta, Gen. Subj. 1996, 1290 (1), 37-45.

(33) Ewers, H.; Helenius, A. Lipid-mediated endocytosis. Cold Spring Harbor Perspect. Biol. 2011, 3 (8), a004721.

(34) Turnbull, W. B.; Precious, B. L.; Homans, S. W. Dissecting the cholera toxin-ganglioside GM1 interaction by isothermal titration calorimetry. J. Am. Chem. Soc. 2004, 126 (4), 1047-1054.

(35) Schon, A.; Freire, E. Thermodynamics of intersubunit interactions in cholera-toxin upon binding to the oligosaccharide portion of its cell-surface receptor, ganglioside-gm1. Biochemistry 1989, 28 (12), 5019-5024.

(36) Taube, S.; Jiang, M. X.; Wobus, C. E. Glycosphingolipids as receptors for non-enveloped viruses. Viruses 2010, 2 (4), 1011-1049.

(37) Karlsson, K. A. Microbial recognition of target-cell glycoconjugates. Curr. Opin. Struct. Biol. 1995, 5 (5), 622-635.
(38) Lee, D. W.; Hsu, H. L.; Bacon, K. B.; Daniel, S. Image restoration and analysis of influenza virions binding to membrane receptors reveal adhesion-strengthening kinetics. PLoS One 2016, 11 (10), e0163437.

(39) Lakshminarayan, R.; Wunder, C.; Becken, U.; Howes, M. T.; Benzing, C.; Arumugam, S.; Sales, S.; Ariotti, N.; Chambon, V.; Lamaze, C.; Loew, D.; Shevchenko, A.; Gaus, K.; Parton, R. G.; Johannes, L. Galectin-3 drives glycosphingolipid-dependent biogenesis of clathrin-independent carriers. Nat. Cell Biol. 2014, 16 (6), 595-603.

(40) Carton, I.; Malinina, L.; Richter, R. P. Dynamic modulation of the glycosphingolipid content in supported lipid bilayers by glycolipid transfer protein. Biophys. J. 2010, 99 (9), 2947-2956.

(41) Sonnino, S.; Mauri, L.; Chigorno, V.; Prinetti, A. Gangliosides as components of lipid membrane domains. Glycobiology 2006, 17 (1), $1 \mathrm{R}-13 \mathrm{R}$.

(42) Bingen, P.; Wang, G.; Steinmetz, N. F.; Rodahl, M.; Richter, R. P. Solvation effects in the quartz crystal microbalance with dissipation monitoring response to biomolecular adsorption. a phenomenological approach. Anal. Chem. 2008, 80 (23), 8880-8890.

(43) Chao, L.; Daniel, S. Measuring the partitioning kinetics of membrane biomolecules using patterned two-phase coexistant lipid bilayers. J. Am. Chem. Soc. 2011, 133 (39), 15635-15643.

(44) Torgersen, M. L.; Skretting, G.; van Deurs, B.; Sandvig, K. Internalization of cholera toxin by different endocytic mechanisms. J. Cell Sci. 2001, 114 (20), 3737-3747.

(45) Baumann, H.; Doyle, D. Turnover of plasma-membrane glycoproteins and glycolipids of hepatoma tissue-culture cells. J. Biol. Chem. 1978, 253 (12), 4408-4418.

(46) Falguieres, T.; Romer, W.; Amessou, M.; Afonso, C.; Wolf, C.; Tabet, J. C.; Lamaze, C.; Johannes, L. Functionally different pools of Shiga toxin receptor, globotriaosyl ceramide, in HeLa cells. FEBS J. 2006, 273 (22), 5205-5218.

(47) Jobling, M. G.; Yang, Z. J.; Kam, W. R.; Lencer, W. I.; Holmes, R. K. A single native ganglioside gm(1)-binding site is sufficient for cholera toxin to bind to cells and complete the intoxication pathway. mBio 2012, 3 (6), e00401-12. 